\title{
Cultura política ciudadana y abstención electoral en el municipio fronterizo de Tijuana
}

\author{
Citizen's political culture and electoral abstention in \\ the border municipality of Tijuana
}

Ana Claudia Coutigno Ramírez ${ }^{\text {* }}$ (i) https://orcid.org/0000-0002-0965-9632

Recibido el 26 de septiembre de 2017.

Aceptado el 12 de enero de 2018.

Publicado el 4 de abril de 2018.

*Autor para correspondencia: Ana Claudia Coutigno Ramírez, correo electrónico: ana.claudia. coutigno@gmail.com

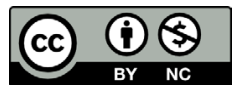

Esta obra está protegida bajo una Licencia Creative Commons Atribución-NoComercial 4.0 Internacional.

\begin{abstract}
${ }^{a}$ El Colegio de la Frontera Norte, Departamento de Estudios de Población, Baja California, México, correo electrónico: ana.claudia.coutigno@gmail.com
\end{abstract}

\section{Resumen:}

El objetivo de este artículo es identificar si la abstención electoral en Tijuana presenta particularidades dada su ubicación fronteriza y el tiempo de residencia de sus ciudadanos, así como las características de su cultura política. Se utilizan dos fuentes para su estudio: a) la estadística electoral en el periodo 1988-2016 para contar con las tendencias de la abstención, y $b$ ) el análisis de los resultados de una encuesta representativa del municipio. Con ellas, se elaboraron índices y una regresión logística para examinar las opiniones ciudadanas acerca del significado del voto, los partidos políticos y su ubicación ideológica. Este estudio es relevante dada la tendencia al incremento de la abstención, siendo ya un problema sustantivo grave para la democracia electoral de la localidad. En la cultura política, los abstencionistas nativos consideran el voto como una forma de expresión respecto del funcionamiento del sistema político y los abstencionistas inmigrantes presentan tendencias diferenciadas acorde al tiempo de residencia.

Palabras clave: abstencionismo, elecciones, cultura política, ciudadanía, ideología.

\section{Abstract:}

The aim of this article is to identify whether the electoral abstention in Tijuana presents particularities given its border location and the time of residence of its citizens, as well as the characteristics of its political culture. Two sources were used for this study: a) electoral statistics for the period 1988-2016 to account for abstention trends, and $b$ ) the analysis of the results of a representative survey of the municipality. With these sources, indices and a logistical regression were developed to examine citizen opinions regarding the meaning of voting, political parties, and their ideological leanings. This study is relevant due to the growing trend towards abstention, which already is a serious and substantive problem for local electoral democracy. In political culture, native abstentionists consider

CÓMO CITAR: Coutigno, A. C. (2018). Cultura política ciudadana y abstención electoral en el municipio fronterizo de Tijuana [Citizen's political culture and electoral abstention in the border municipality of Tijuana]. Estudios Fronterizos, 19, e007. https://doi. org/10.21670/ref.1807007 
voting as a form of expression regarding the functioning of the political system, and immigrant abstentionists show differential tendencies according to residence time.

Keywords: electoral abstention, elections, political culture, citizenship, ideology.

\section{Introducción}

El politólogo Dieter Nohlen (2004) señala que en algunos países de América Latina es muy notorio dentro del discurso político impulsar la participación de los ciudadanos en los procesos electorales. Indica que el supuesto subyacente es que la democracia se legitima y al mismo tiempo se consolida a través de una alta participación electoral, siendo esta la forma de participación política más importante en un régimen democrático por tres razones principales: a) porque incluye la mayor cantidad de ciudadanos y al mismo tiempo garantiza la participación más igualitaria de los miembros de una sociedad; $b$ ) por ser el canal central que vincula al electorado y a sus preferencias políticas con el poder, el cual se manifiesta en la elección de sus representantes; y $c$ ) porque a través de esta vinculación, toda la sociedad se relaciona o es afectada por el resultado, el cual se materializa en la legislación como parte de las políticas públicas generadas.

Si la participación electoral representa una expectativa fundamental, resulta necesario indagar por qué algunos ciudadanos deciden dejar de emitir su voto. La abstención, durante el proceso electoral, se manifiesta como una expresión que teóricamente es contradictoria a los principios democráticos, actitud política que no es considerada beneficiosa para la construcción de ciudadanía, que tiene como eje central a la participación ciudadana (Sartori, 2007).

En la actualidad, la abstención electoral es un tema que se discute constantemente en el marco de la democracia procedimental contemporánea. Esta última señala un conjunto de principios que determinan el modo en que los ciudadanos se deben de conducir bajo un régimen democrático. Estos principios se utilizan también como supuestos que orientan los procesos democráticos y el rol que desempeña el ciudadano en su desarrollo, así como la capacidad de este para evaluar su calidad. Dicha evaluación es emitida el día de los comicios, donde se valora en cierta media a la saliente administración, así como en la elección de sus nuevos representantes, ya sea al ratificar al mismo partido político, alternando su preferencia partidaria o alejándose de las urnas.

En este sentido, Merino (1995) dice que el porcentaje de participación electoral depende, por un lado, de encontrar el equilibrio entre las razones que animan a los ciudadanos a participar y sus posibilidades reales para hacerlo, y por el otro, de su voluntad para intervenir activamente en los asuntos públicos; y aunque existan las mejores condiciones en el procedimiento y un buen entorno político, al final siempre habrá quien encuentre más razones para abstenerse que para participar.

Considerando lo anterior, en este artículo se estudia la abstención electoral en el municipio fronterizo de Tijuana ubicado en el norte del estado de Baja California, y cuya participación electoral es relevante dada la tendencia al incremento en los porcentajes de abstención, tanto en elecciones locales como federales, durante el periodo que va de 1988 a 2016. Basta señalar que en la última elección local correspondiente a 2016, en promedio, la abstención alcanzó $70 \%$ sobre el listado nominal. 
Un punto de partida sin el cual no se puede analizar dicha conducta es el contexto en el cual se convive de manera cotidiana. En los últimos estudios del Instituto Nacional Electoral (INE) y de El Colegio de México se confirma que "la participación política no solo refleja diferencias en términos socioeconómicos o incluso actitudes de los ciudadanos; la participación política también se ve influida por el contexto social, económico y político en el que los ciudadanos se encuentran" (Somuano, Nieto y Zaremberg, 2014, p. 22). Este estudio parte de tres supuestos: El primero, es que además de las características individuales del ciudadano (perfil socioeconómico) y de su actitud frente a la política, la participación política es influida por el contexto. El segundo, que las características económicas, políticas y sociales del estado afectan los niveles de participación ciudadana. Y tercero, que las características de los ciudadanos y actitudes frente a la política son diferentes en cada estado debido a su contexto (Somuano et al., 2014). Estos tres supuestos son fundamentales también para analizar el alejamiento de las urnas por parte del ciudadano, en el contexto de una ciudad fronteriza del norte de México, como es Tijuana.

En atención a ello, es importante, en primer lugar, considerar las dinámicas demográficas y económicas de la ciudad, propias de una zona colindante con Estados Unidos. Y, en segundo lugar, sus particularidades históricas y políticas, principalmente su juventud y su desarrollo bajo políticas económicas y sociales dictadas desde el centro de México.

Respecto a la dinámica demográfica de Tijuana, esta es impactada por la inmigración. La cual ha sido el eje principal para su desarrollo económico y social desde principios del siglo $\mathrm{xx}$ hasta la actualidad; existiendo en este municipio un crisol de perspectivas ciudadanas hacia la política en general y hacia la participación electoral, bajo el supuesto antes señalado.

Para precisar este tema, son útiles los estudios de Zenteno (1995), quien identifica características muy claras en el crecimiento de Tijuana, a través de grandes olas de inmigración. Señala que esta ciudad es el claro ejemplo de una historia contemporánea caracterizada por una fuerte dependencia con Estados Unidos y una nula dependencia con el resto de México en sus primeros años de desarrollo (1920-1930). En este sentido, el vaivén económico de las siguientes décadas en la Unión Americana impactó en los flujos migratorios en dos sentidos: en demanda de trabajadores mexicanos, así como la deportación de los mismos, aspectos que han definido la dinámica económica y social del municipio hasta la actualidad. Un dato significativo, es que Estados Unidos fue, es y seguirá siendo un fuerte motor en la demanda de trabajadores, por lo tanto, Tijuana se convierte en un centro fronterizo e "importante lugar de espera para insertarse laboralmente en la economía estadunidense" (Zenteno, 1995, p. 130). Sin embargo, no todos logran cruzar la línea fronteriza y finalmente se quedan en Tijuana.

Desde otro ángulo, es a partir de la presidencia de Lázaro Cárdenas (1934-1940) que se implementan políticas públicas que detonan económicamente la zona fronteriza del norte de México y con ello inicia un nuevo contexto social, económico y político en la región, particularmente en el municipio de Tijuana, con mejoras en infraestructura y medios de comunicación lo que también atrajo a numerosas corrientes migratorias en busca de un mercado laboral con mayores ventajas económicas (Zenteno, 1995). Ello se reflejó en altas tasas de crecimiento demográfico para el municipio. Por ejemplo, desde 1940 hasta principios de 1970, Tijuana superó las tasas de crecimiento a nivel nacional e incluso de los demás municipios fronterizos. En la década de 1970 se presenta una pequeña disminución, recuperándose este indicador en la década 
de 1980 (Zenteno, 1995). Finalmente, durante la década de 1990 hubo una tasa de crecimiento demográfico de $5 \%$ y $2.5 \%$ en la década 2000-2010 (Consejo Nacional de Población [Conapo], 2012), lo que sigue contribuyendo al repunte de la inmigración y por consecuencia al crecimiento demográfico.

Las preguntas centrales de este estudio son las siguientes: ¿La abstención electoral en Tijuana, presenta particularidades derivadas de su ubicación fronteriza? ¿La abstención electoral muestra patrones de comportamiento diferenciados según el tiempo de residencia de sus habitantes?

Sobre estas preguntas, consideremos que dicha abstención tiene dos particularidades en la cultura política de los ciudadanos fronterizos, de acuerdo con nuestra investigación empírica. En primer lugar, se encuentra la existencia de una abstención electoral involuntaria debido a factores técnico-administrativos e institucionales, por ejemplo, en la necesidad de realizar un trámite de actualización de domicilio en el listado nominal para los inmigrantes de reciente arribo; y, en segundo lugar, está la abstención electoral voluntaria. Este último tipo de abstención electoral tiene dos vertientes, la primera es la abstención social o individual, en donde se inserta el concepto de apropiación de ciudad ${ }^{1}$ y lo transitorio de la vida fronteriza. La segunda, es una actitud que engloba una forma de expresión compleja que utiliza el ciudadano para hacer explícita su valoración negativa a una parte del desarrollo del régimen democrático, el cual se manifiesta en: a) la falta de confianza que se tiene hacia los candidatos propuestos por los partidos políticos en los comicios electorales; $b$ ) el desencanto derivado del incumplimiento por parte de los partidos políticos de promesas electorales subsecuentes; y $c$ ) la falta de reciprocidad democrática de los gobiernos municipales en la solución de los problemas locales (Coutigno, 2012).

Hechas las consideraciones anteriores, en la primera parte se analizan las estadísticas electorales del municipio de Tijuana con el objetivo de identificar y confirmar la tendencia hacia el incremento de la abstención electoral, así como su concentración, independientemente de tratarse de una elección federal o local; asimismo, identificar si con esta tendencia se puede calificar a la abstención en Tijuana como una problemática real.

En una segunda parte, se presenta un primer acercamiento a la cultura política del ciudadano elector residente en el municipio de Tijuana, a través de la generación de índices para identificar las relaciones significativas en torno a la abstención electoral. Los datos que se utilizan para su elaboración son exclusivos de la Encuesta sobre cultura política y conducta electoral de los ciudadanos residentes en el municipio de Tijuana, Baja California, febrero 2010. ${ }^{2}$ En una tercera parte se hace uso de un modelo de

\footnotetext{
${ }^{1}$ El grado de apropiación de la ciudad es retomado del estudio de Acosta, Solís y Alonso (2012) quienes consideraron tres dimensiones: el nivel de compromiso con la ciudad, la intensidad de las relaciones sociales en la ciudad y la intensidad del uso del espacio público.

${ }^{2}$ La encuesta se realizó para efectos de esta investigación por medio de Berumen y Asociados, S.A. de C.V. del 27 de febrero al 1 de marzo de 2010, periodo que antecede a las precampañas electorales que iniciaron el 12 de marzo del mismo año, con el objetivo de que estas no afectaran la opinión de los electores. Se aplicó a 300 personas residentes en igual número de hogares, mayores de 18 años que contaban con credencial para votar domiciliada en el municipio de Tijuana. Se seleccionó una muestra probabilística de 60 secciones electorales del municipio de Tijuana, utilizando como marco muestral el listado de secciones electorales y su padrón electoral según datos del INE para febrero de 2010. Las secciones electorales se seleccionaron con probabilidad proporcional al tamaño de su padrón electoral. De cada sección incluida en la muestra se seleccionó aleatoriamente una manzana por sección, y de cada una de ellas se seleccionaron alrededor de siete viviendas para obtener cinco entrevistas por sección electoral.
} 
regresión logística con los datos de la misma encuesta, para estimar los elementos sociodemográficos asociados a la probabilidad de que una persona sea abstencionista. En el cuarto apartado se presentan las conclusiones y algunas reflexiones finales.

\section{Niveles y tendencias de la abstención}

El concepto de abstención electoral utilizado en este documento es definido como la no participación en el acto de votar de quienes tienen derecho a ello y es, a la vez, un indicador que muestra el porcentaje de los no votantes sobre el total de los que tienen derecho al voto (Alcubilla, s.f.). En el caso de este estudio, para medir la abstención se toma como base a los ciudadanos que están registrados en el listado nominal y no con base al padrón electoral ni sobre la población en general que tiene el derecho al sufragio, ya que ello conduce a otro resultado y principalmente a otra interpretación.

La estadística electoral para el municipio de Tijuana se presenta de manera general en la Figura 1. En ella se observa el resultado de la abstención durante el periodo de 1988 a 2016. Y como se observa, es a partir del proceso electoral de 1997 que se identifica la clara tendencia al incremento de la abstención. Los datos indican que al final del periodo de análisis (elección 2016) votaron solo tres de cada diez ciudadanos, dato que sugiere la construcción de un proceso de separación entre el ciudadano y la democracia electoral.

Figura 1: Tendencia de la abstención política electoral en Tijuana, Baja California, 1988-2016

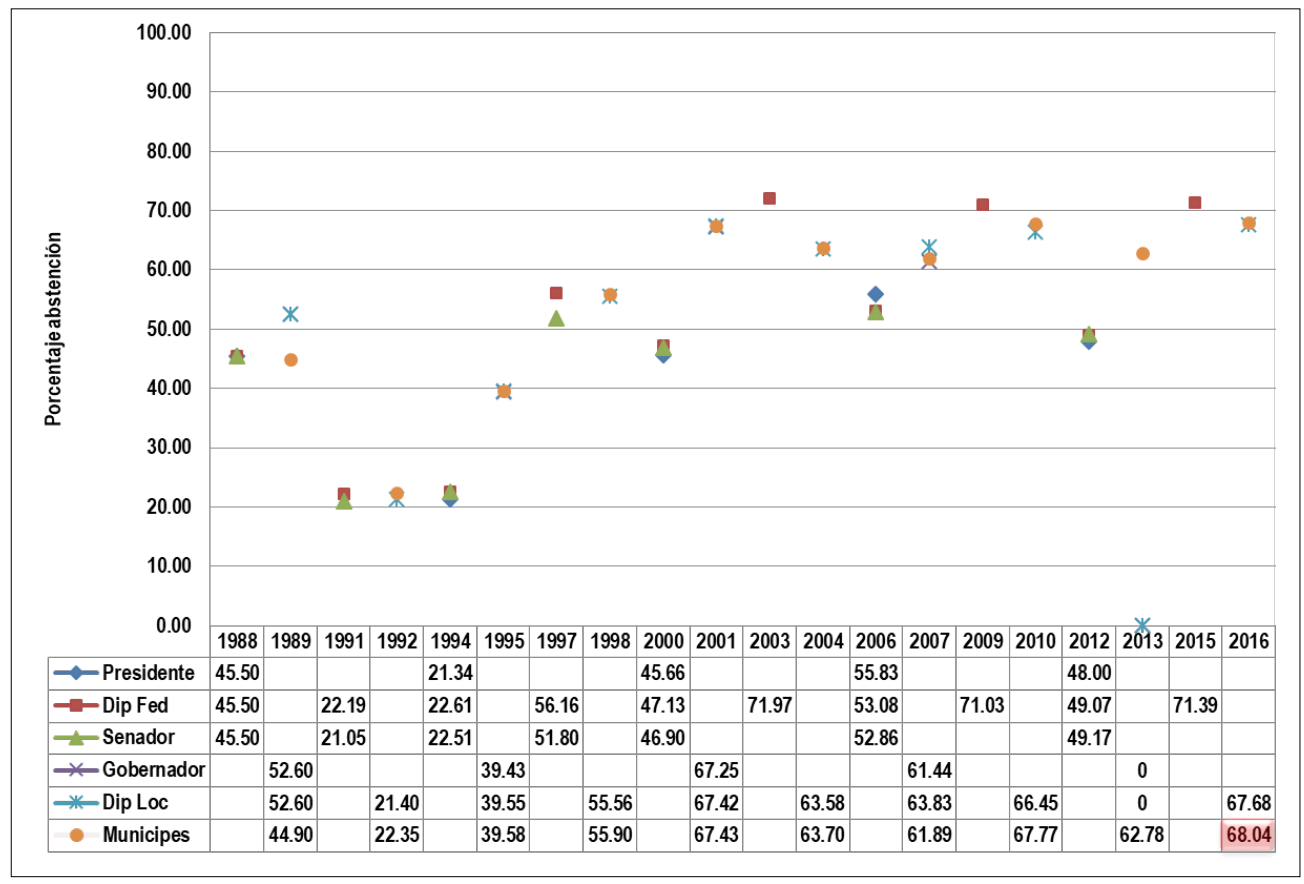

Fuente: Elaboración propia con información de Instituto Estatal Electoral de Baja California (IEEBC, 2016) e INE (2016). 
En suma, a partir del análisis de los resultados electorales, se puede considerar que los ciudadanos que residen en el municipio de Tijuana se están alejando de las urnas, independientemente del tipo de proceso electoral. Ello muestra la complejidad de la conducta electoral de estos ciudadanos, aún si solo se toma el dato estadístico. Las preguntas obligadas en este momento son: ¿Se podría considerar ya un problema estructural o sustantivo la abstención electoral? ¿Qué factor o factores están incidiendo en los ciudadanos fronterizos que los lleva a no ejercer este derecho? ¿Por qué su incremento de manera constante en las elecciones locales? ¿Qué efectos tendrá en la democracia electoral y en la representación política si continúa esta tendencia sistemática de la abstención?

Un primer punto de reflexión para argumentar de manera general las causas de la tendencia oscilatoria de la participación electoral de los tijuanenses es que, de acuerdo con lo que señalan los estudios sobre el diseño institucional del sistema electoral, la simultaneidad o concurrencia en las elecciones impacta de manera significativa en una mayor participación electoral, independientemente de la convocatoria de los partidos políticos y más si es una elección presidencial (Espinoza, 2007; Gómez, 2009, p. 88; Nohlen, 2003). Para el caso de las elecciones en el estado de Baja California, las elecciones no son concurrentes con las federales, por lo que este punto debe ponderarse, pero no como una determinante.

Tomando en cuenta que la participación electoral disminuye en las elecciones intermedias para diputados federales, pudiera parecer normal el incremento de la abstención ocurrida en Tijuana en 2003, 2009 y 2015 que alcanza más de 70\%; siendo en 2015, cuando se presenta la abstención más alta a nivel nacional en el conjunto de municipios. Además, como se observa también en la Figura 1, con respecto a las elecciones para el ejecutivo federal de 2006 y 2012, los resultados indican una disminución de $7.8 \%$ de la abstención de una elección a otra, efecto de una elección presidencial, como se ha mencionado en párrafos anteriores. Sin embargo, al analizar las elecciones locales de 2001, 2004, 2007, 2010, 2013 y 2016, la abstención frente a las elecciones presidenciales es en promedio mayor por 10 puntos porcentuales, independientemente de que haya o no una elección para gobernador.

Para analizar con más detenimiento la abstención en Tijuana, hemos seleccionado los comicios para presidentes municipales de 1989 a 2016, porque este fenómeno político representa la elección de una autoridad local que de alguna manera está cerca de la ciudadanía, que identifica y resuelve los principales problemas que afectan directamente al municipio, por lo que se esperaría que los ciudadanos estén más atentos a sus propuestas de campaña y al proceso electoral en general. Sin embargo, los datos estadísticos electorales nos indican que no es así.

En los resultados estadísticos que se presentan en la Figura 2, se observa una clara tendencia al incremento de la abstención de las elecciones en Tijuana de 1989 a 2016, siendo 2016 el punto más alto. Es importante señalar que con este análisis longitudinal se hace evidente que las autoridades municipales solo son elegidas por tres de cada diez ciudadanos. Lo que hace cuestionarnos, con mucha razón, cuál es el límite de legitimidad para este ejercicio electoral. 
Figura 2: Porcentaje de abstención en las elecciones para presidente municipal en Tijuana, Baja California, 1989-2016

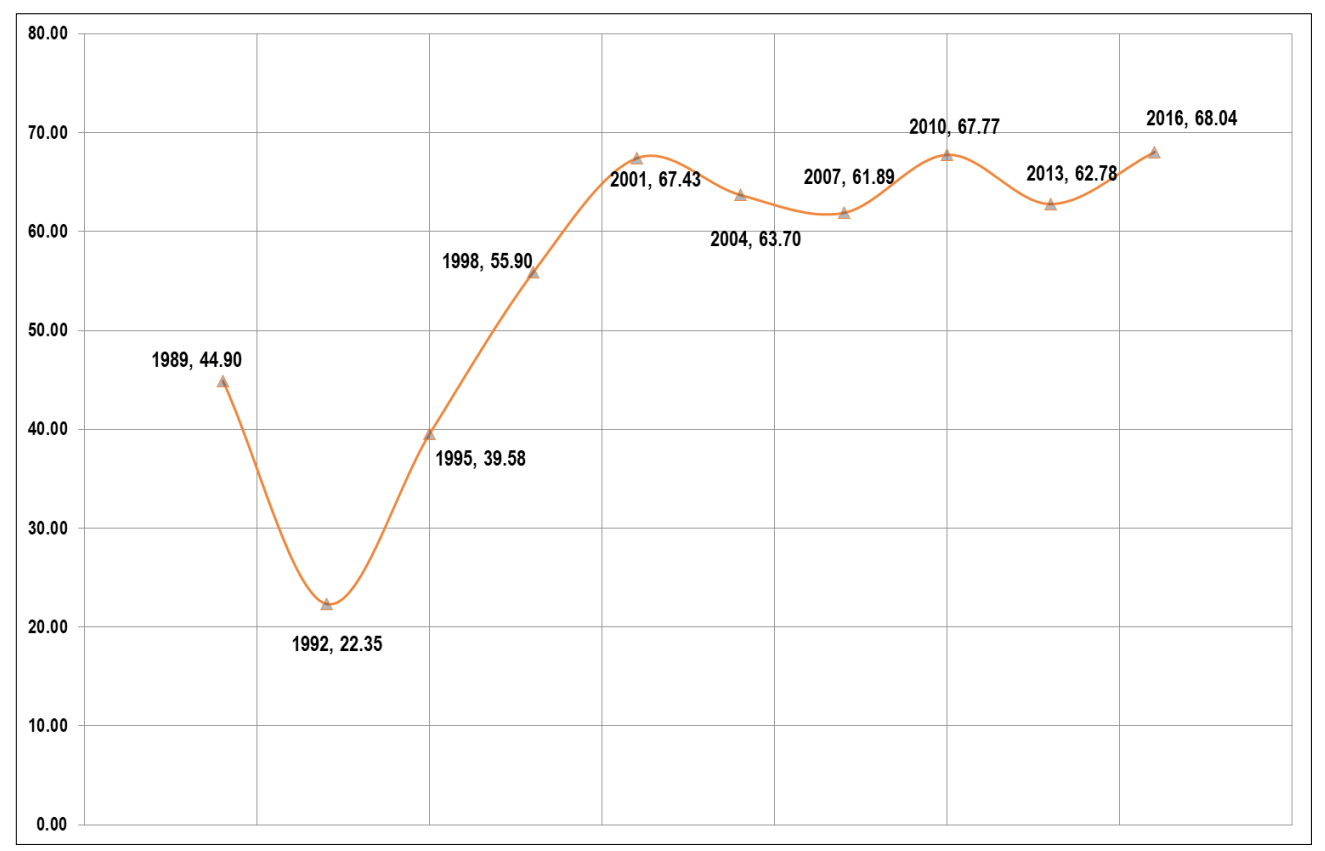

Fuente: Elaboración propia con información de IEEBC (2016).

Dicho lo anterior, se podría considerar que en la actualidad la abstención electoral puede considerarse como un problema sustantivo en el municipio de Tijuana. Ahora bien, si llevamos nuestro análisis a la dimensión territorial indicada por Raventós (2003), es decir, que si la tendencia de abstención es sistemática ya puede ser considerada problemática en sí misma, pero cuando tiende a concentrarse en sectores o espacios específicos de la población, como en la población más pobre o de menor nivel educativo, en personas que viven en lugares remotos, en la población indígena o en un género en particular, estamos entrando en una exclusión social o política. Además, menciona que el riesgo que conlleva este tipo de abstención localizada y sistemática es la definición de perfiles de quien determina los resultados electorales, pudiéndose inclinar hacia formas de centralización elitistas.

La concentración de la abstención electoral es pues un problema serio para los sistemas democráticos electorales que merece un análisis más preciso. Por ello resulta sustantivo localizar a los ciudadanos que residen en el municipio de Tijuana y que deciden no votar bajo el esquema de su ubicación por distritos y secciones electorales. Como hemos señalado en párrafos anteriores, la abstención asciende a más de $68 \%$ en elecciones locales, y es superior a $70 \%$ en la elección del Congreso Federal. El análisis espacial nos permitirá confirmar si existe concentración o no de la abstención en el municipio, y saber si ya estamos entrando a un problema sustantivo en nuestro sistema electoral democrático en la región fronteriza. Para ello utilizaremos el recurso analítico de la localización geográfica y sistemática de los resultados de la abstención por distrito electoral y sección electoral de 2004 a 2016.

En relación con este tema, el municipio de Tijuana está dividido, a nivel federal, en cuatro distritos $(4,5,6$ y 8$)$ y para las elecciones locales se divide en siete distritos 
(VIII, IX, X, XI, XII, XIII y XVI). Para ambas divisiones le corresponden un total de 758 secciones electorales, ${ }^{3}$ las cuales tienen la ventaja de ser las mismas tanto para el ámbito federal como local.

Para un primer acercamiento al análisis de la abstención a nivel sección electoral, se han reagrupado las secciones de acuerdo con el porcentaje de abstención reportado en las estadísticas, y por el distrito que le corresponde a nivel local. Posterior a ello, se observa el comportamiento de la abstención en las secciones y se identifican las que mantienen el promedio más alto de abstención y su oscilación en el tiempo. Del resultado empírico, se concluye que la abstención electoral presenta una tendencia diferenciada en cuanto a su intensidad en este nivel y se propone la siguiente clasificación para los distritos electorales (ver Tabla 1).

Tabla 1: Clasificación de la tendencia de abstención electoral en el municipio de Tijuana, Baja California, 2004-2016

\begin{tabular}{|l|l|}
\hline Clasificación & \multicolumn{1}{c}{ Tendencia de la abstención } \\
\hline Grupo A & Con una abstención constante con incrementos sustanciales. \\
\hline Grupo B & Con una abstención constante con incrementos no tan sustanciales. \\
\hline Grupo C & Cambia de participación a abstención sustancial coyuntural. \\
\hline
\end{tabular}

Fuente: Elaboración propia con información de IEEBC (2016).

Así pues, con la aplicación de este recurso analítico, se identificó la concentración de la abstención en los distritos electorales quedando de la siguiente manera: en el Grupo A, se encuentra el distrito XII que representa un área político-electoral con una presencia histórica de la abstención y con una marcada tendencia creciente en este fenómeno; en el Grupo B, se concentran los distritos electorales XI, XIII y XVI, cuyo rasgo característico es la estabilidad en el patrón de abstencionistas contantes que se mantiene con incrementos no tan sustanciales; finalmente, en el Grupo C, se encuentran los distritos VIII, IX, y x, cuya distinción es la emergencia coyuntural (en la última elección de 2013) de un alto índice de abstención, a pesar de haber sido distritos con una participación electoral relativamente alta respecto a las demás elecciones.

En suma, es el distrito xiI del municipio de Tijuana la zona con mayor exclusión social y política dada su concentración e incremento del número de ciudadanos que han decidido no ejercer su derecho al voto, de acuerdo con Raventós (2003). El siguiente paso sería identificar su perfil sociodemográfico, por presentarse en posteriores investigaciones.

\footnotetext{
3 Metodológicamente, una de las ventajas del estudio a nivel sección electoral es que nos permite estudiar tanto los comicios locales como los federales, ya que son las mismas secciones en los dos niveles. Sin embargo, en un estudio longitudinal de esta naturaleza se tienen que controlar los procesos de redistritación, la creación de nuevas secciones, inclusive la fusión entre secciones y la desaparición de otras. Para este análisis se tomaron solo aquellas secciones que en el tiempo se han mantenido sin cambio.
} 


\section{Cultura política y perfil del abstencionista}

Con los datos anteriores en mente, y para un acercamiento más al pensamiento del ciudadano frente al sistema democrático, el objetivo de esta sección es conocer la cultura política del ciudadano elector que reside en el municipio de Tijuana. En particular, se busca identificar las relaciones significativas en torno a la abstención electoral y la cultura política, entendida esta última como "los valores, concepciones y actitudes que se orientan hacia el ámbito específicamente político. Es decir, el conjunto de elementos que configuran la percepción subjetiva que tiene una población respecto al poder" (Peschard, 2001, p. 3), considerando al elector como "la persona que reúne las condiciones exigidas por la Constitución o las leyes, para ejercitar el derecho de sufragio y que, por tanto, tiene facultades para influir con su voto en la elección o nombramiento de concejales, diputados, senadores e incluso del jefe del Estado" (Cabanellas, citado en Barquín, 2003, p. 163).

Es así que el elector constituye la unidad de análisis básica, pues a través de él se puede estudiar al ciudadano que reside en Tijuana y su cultura política. Los datos de este estudio parten en exclusiva de la Encuesta sobre cultura política y conducta electoral de febrero 2010. La ruta analítica que seguiremos es, primeramente, la descripción del perfil sociodemográfico del abstencionista; y en segundo, la presentación de cuatro índices que se elaboraron para distinguir y ponderar la cultura política del elector que vota y del elector abstencionista. Sumado a ello se incluye, como vector analítico, el tiempo que tiene de residir el ciudadano, ${ }^{4}$ como factor que puede marcar la diferencia en su actitud política.

En lo que se refiere a las características sociodemográficas del grupo de abstencionistas, estos muestran un porcentaje de abstención electoral muy similar entre mujeres $(50.8 \%)$ y hombres $(49.2 \%)$. Y respecto a su distribución por edad, poco más de dos terceras partes de ellos se ubican en el rango de los 30 a 44 años, con un porcentaje relativamente bajo en los grupos extremos de edad. $\mathrm{Al}$ analizar lo relacionado con la edad y el sexo, en el caso de las mujeres, $66 \%$ de las abstencionistas se ubica entre las edades 35 a 44 años; en tanto que, en el caso de los hombres, tres de cada cuatro abstencionistas se ubican entre los 25 y 44 años de edad.

En este mismo perfil, una variable significativa es el nivel de escolaridad, la cual, ha sido ampliamente discutida en la academia como una variable que interviene en el comportamiento electoral del ciudadano. En estos términos, seis de cada diez abstencionistas que residen en el municipio de Tijuana cursaron la educación básica como máximo nivel de estudios. Sin embargo, es preciso señalar que hay un grupo significativo de abstencionistas que cuentan con estudios técnicos y otros con estudios de preparatoria. En relación con la actividad principal que tiene este grupo de electores, los datos de la encuesta revelan que en su gran mayoría se trata de una población que trabaja; este porcentaje es más marcado en el caso de los hombres; en tanto que las mujeres, una cuarta parte de ellas se dedica a quehaceres del hogar. Vale la pena señalar que, en términos de su condición migratoria, de los abstencionistas, $53.8 \%$ son nativos del municipio y el restante $46.2 \%$ nacieron en otro estado diferente al de Baja California, lo que se examinará a mayor detalle en párrafos siguientes.

\footnotetext{
${ }^{4}$ Los ciudadanos entrevistados contaban al momento de la entrevista con credencial para votar expedida en Baja California.
} 
Respecto a los cuatro indicadores estadísticos que nos permitirán ponderar la cultura política de los electores que residen en el municipio, ellos se construyeron de la siguiente manera:

a) Índice de ubicación ideológica: Para la integración de este índice, el propio ciudadano se autoubica ideológicamente en una escala de valores que van del 1 al 10, en donde del 1 al 4 se está a la izquierda, 5 al centro y del 6 al 10 a la derecha. Posterior a ello, se estandarizaron los resultados y se construyeron tres categorías de la variable: izquierda, centro y derecha.

b) Índice de participación ciudadana: Este índice mide el grado de participación ciudadana de los electores en 13 diferentes organizaciones, como las vecinales y la asociación de padres de familia, entre otras. Las respuestas y su resultado se dividen en el total de organizaciones analizadas para elaborar tres categorías de análisis: ciudadanos sin participación, ciudadanos con participación y ciudadanos con alta participación.

c) Índice de confianza institucional: Con este índice se trata de medir el grado de confianza que los ciudadanos tienen en 19 organizaciones. A partir de esta información se construyen cuatro categorías de análisis: sin confianza, confianza baja, confianza media y confianza alta.

d) Índice de participación electoral: Uno de los aspectos importantes en el comportamiento electoral que se identificó en este estudio es la presencia de tres tipos de electores: los que votan, los abstencionistas constantes, y los que en algunas elecciones votan y en otras se abstienen (itinerantes). Quizás el comportamiento de este último elector tenga que ver con factores relativos al sistema político en una dimensión individual y coyuntural. Con los resultados anteriores se construyó el índice de participación electoral con tres categorías de análisis: participantes, abstencionistas itinerantes y los abstencionistas constantes.

La primera relación de estos índices es su ubicación ideológica ya que de acuerdo con la idea de Alcántara (2008), los conceptos de izquierda y derecha son componentes simplificadores del conflicto político y juegan un papel orientador a la hora de tipificar las distintas orientaciones políticas por parte de los electores. En este sentido, en la Tabla 2 se puede observar el tiempo de residencia en Tijuana y la autoubicación ideológica. De manera general los inmigrantes se ubican en el centro ideológico. Aquellos con entre uno y cinco años de residencia, se ubican más en la derecha $(24.8 \%)$ y menos en la izquierda $(23.7 \%)$, mientras que los que tienen más de 11 años radicando en Tijuana, aumentan su ubicación en la izquierda (28\%), casi alcanzando a los nativos del estado (31\%). En la simpatía por algún partido político se observan diferencias importantes dependiendo el tiempo de residencia, la primera es que quienes cuentan con uno a diez años de residencia simpatizan más con el Partido Revolucionario Institucional (PRI) y de aquellos con 11 años en adelante, 53\% simpatiza con el Partido Acción Nacional (PAN), con un porcentaje más alto que los nativos $(35.9 \%)$. 
Tabla 2: Índice de ubicación ideológica y simpatía por algún partido político según tiempo de residencia de los electores en el municipio de Tijuana, Baja California, 2010.

Distribución porcentual

\begin{tabular}{|c|c|c|c|c|c|}
\hline \multirow{2}{*}{ Tiempo de residencia } & \multicolumn{5}{|c|}{ Ubicación ideológica } \\
\hline & Izquierda & Centro & & recha & Total \\
\hline Nativo & 31.0 & 59.8 & & 9.2 & 100.0 \\
\hline 01 a 05 & 23.7 & 51.5 & & 4.8 & 100.0 \\
\hline 06 a 10 & 26.2 & 65.8 & & 3.0 & 100.0 \\
\hline \multirow[t]{3}{*}{11 en adelante } & 28.0 & 66.7 & & 5.3 & 100.0 \\
\hline & \multicolumn{5}{|c|}{ Simpatía por algún partido } \\
\hline & PRD & PRI & PAN & Otro/ninguno & Total \\
\hline Nativo & 6.4 & 27.0 & 35.9 & 30.7 & 100.0 \\
\hline 01 a 05 & 23.2 & 48.1 & 6.9 & 21.8 & 100.0 \\
\hline 06 a 10 & 1.5 & 46.0 & 26.6 & 25.9 & 100.0 \\
\hline 11 en adelante & 6.2 & 15.1 & 53.0 & 25.7 & 100.0 \\
\hline
\end{tabular}

Fuente: Elaboración propia con base en los datos de la Encuesta sobre cultura política y conducta electoral de los ciudadanos residentes en el municipio de Tijuana, Baja California, febrero 2010.

La combinación entre el índice de participación ciudadana y el de ubicación ideológica (Tabla 3), nos señala que la derecha (ideológica), tiene dos opciones fuertes: participan electoralmente $(73.4 \%)$ o son abstencionistas itinerantes $(24.8 \%)$, pues los abstencionistas constantes son muy pocos (1.8\%). Los que se autoubican en la izquierda participan electoralmente un poco más que los de la derecha $(75.6 \%)$, pero su porcentaje de abstencionistas constantes se incrementa en siete puntos porcentuales respecto a los de la derecha. Los que se autoubican en el centro ideológico son más abstencionistas constantes que los de izquierda y derecha (10.2\%).

Tabla 3: Índice de ubicación ideológica e índice de participación electoral de los electores en el municipio de Tijuana, Baja California, 2010. Distribución porcentual

\begin{tabular}{|c|c|c|c|c|}
\hline \multirow{2}{*}{$\begin{array}{l}\text { Ubicación } \\
\text { ideológica }\end{array}$} & \multicolumn{4}{|c|}{ Índice de participación electoral } \\
\hline & Participa & $\begin{array}{l}\text { Abstencionista } \\
\text { itinerante }\end{array}$ & $\begin{array}{l}\text { Abstencionista } \\
\text { constante }\end{array}$ & Total \\
\hline Izquierda & 75.6 & 15.4 & 8.9 & 100.0 \\
\hline Centro & 76.5 & 13.4 & 10.2 & 100.0 \\
\hline Derecha & 73.4 & 24.8 & 1.8 & 100.0 \\
\hline Total & 75.9 & 15.1 & 9.0 & 100.0 \\
\hline
\end{tabular}

Fuente: Elaboración propia con base en los datos de la Encuesta sobre cultura política y conducta electoral de los ciudadanos residentes en el municipio de Tijuana, Baja California, febrero 2010. 
De igual forma, el índice de participación ciudadana ofrece una información muy rica para identificar diferentes formas de intervenir en el ámbito público. Un aspecto que es importante discutir en la participación de los migrantes, que se retoma de los estudios de Acosta et al. (2012), es la relación de los habitantes con el territorio (que llamaremos también contexto). Para el municipio de Tijuana, este contexto está mediado por lo transitorio debido a su ubicación fronteriza y es puente para insertarse en el mercado laboral de Estados Unidos, como se ha analizado. Ello puede implicar "bajos grados de apropiación de la ciudad y en que, a pesar del desarrollo económico y social alcanzado en estas, los habitantes perciben rezagos en aspectos relacionados con el entorno urbano" (Acosta et al., 2012, p. 11). Sumado a ello, retomando a Castells y Cusminsky (citados en Acosta et al., 2012, p. 11), indican que esta relación difusa, precaria y fragmentada que tienen los ciudadanos que habitan en la ciudad fronteriza, puede implicar un impacto negativo en la calidad de vida, ya que estos factores son fundamentales para generar ciudadanía e impulsar un compromiso con la comunidad en que se reside y "que, para habitar con 'calidad', exige sus derechos y cumple con sus obligaciones" (Acosta et al., 2012, p. 11).

Después de mencionar esto, presentamos los resultados de la participación ciudadana de los electores, de acuerdo con su residencia en el municipio. En primer lugar, los ciudadanos que tienen de uno a cinco años de residencia, tienen una alta participación ciudadana (36.27\%), y de seis años en adelante disminuye de manera sustantiva su participación en la esfera pública, menor a diez por ciento (sumando con participación alta y con participación). Los porcentajes sin participación superan a los ciudadanos nativos. Esta información puede estar asociada al grado de apropiación de la ciudad, como se había mencionado (ver Figura 3).

Figura 3: Participación política según tiempo de residencia de los electores en el municipio de Tijuana, Baja California, 2010. Distribución porcentual

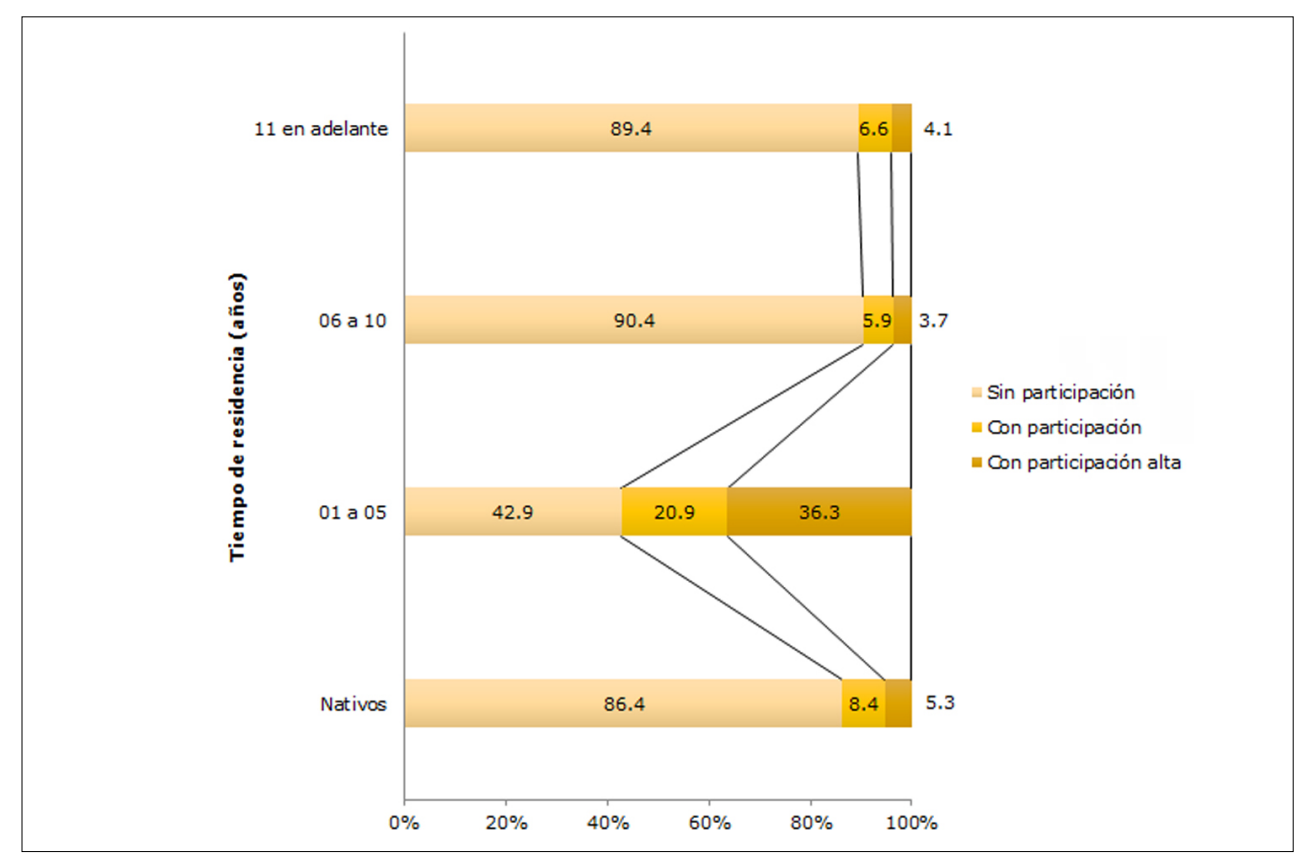

Fuente: Elaboración propia con base en los datos de la Encuesta sobre cultura política y conducta electoral de los ciudadanos residentes en el municipio de Tijuana, Baja California, febrero 2010. 
Sumado al análisis anterior, combinamos ahora el índice de participación ciudadana y el índice de participación electoral. El resultado de ello, es que los electores que no participan en ninguna organización, pero sí lo hacen en los procesos electorales, ascienden a $75.2 \%$. En este sentido, si bien este grupo emite su voto, su participación se queda hasta este punto, permaneciendo al margen de otra forma de participación en los asuntos públicos y delega las responsabilidades y toma de decisiones al gobierno que obtiene la mayoría de los votos. En esta misma categoría están los ciudadanos que deciden no participar en los procesos electorales y además no participan en ningún otro tipo de actividad de interés público o social, por lo que pudieran denominarse como indiferentes o alejados totalmente de los asuntos de interés público, representando $10.2 \%$ como se muestra en la Tabla 4 .

Tabla 4: Índice de participación ciudadana e índice de participación electoral de los electores en el municipio de Tijuana, Baja California, 2010. Distribución porcentual

\begin{tabular}{|c|c|c|c|c|}
\hline \multirow{2}{*}{$\begin{array}{c}\text { Participación } \\
\text { ciudadana }\end{array}$} & \multicolumn{4}{|c|}{ Índice de participación electoral } \\
\hline & $\begin{array}{c}\text { Participación } \\
\text { electoral }\end{array}$ & $\begin{array}{l}\text { Abstencionista } \\
\text { itinerante }\end{array}$ & $\begin{array}{l}\text { Abstencionista } \\
\text { constante }\end{array}$ & Total \\
\hline Sin participación & 75.2 & 14.6 & 10.2 & 100.0 \\
\hline Con participación & 92.9 & 6.0 & 1.1 & 100.0 \\
\hline Con participación alta & 62.7 & 32.8 & 4.5 & 100.0 \\
\hline Total & 75.9 & 15.1 & 9.0 & 100.0 \\
\hline
\end{tabular}

Fuente: Elaboración propia con base en los datos de la Encuesta sobre cultura política y conducta electoral de los ciudadanos residentes en el municipio de Tijuana, Baja California, febrero 2010.

El grupo que emite su voto, pero a su vez participa en los asuntos públicos a través de diferentes organizaciones, ya sea las que impulsan el gobierno o las que se derivan de las organizaciones sociales, representa 92.9\%. Los miembros de este grupo podrían ser denominados como ciudadanos activos en la democracia. El grupo de ciudadanos con participación alta merece una atención especial, ya que solo $62.7 \%$ es constante en su participación electoral y $32.8 \%$ es abstencionista itinerante, esto último pudiera referirnos de alguna manera a circunstancias coyunturales que lo hacen participar de forma diferencial electoralmente.

Es importante considerar también al grupo de ciudadanos que, si bien no participa en los comicios electorales, se involucra a través de otras formas de intervención en los asuntos públicos, en este caso en algún tipo de organización, y ahí es donde prefieren hacer efectiva su participación en la democracia; $4.5 \%$ reportó ser de este tipo de elector (Tabla 4).

Un aspecto muy relacionado con el comportamiento electoral es la confianza en las instituciones, este es uno de los temas que más se ha discutido por el impacto que ha tenido en México en diversos foros y en encuestas a nivel nacional. Además de acuerdo con Salazar y Temkin (2007), la confianza es una variable que interviene en los juicios subjetivos que los electores hacen de sus instituciones como respuesta a la incertidumbre inherente a los procesos democráticos. En general, se puede decir que existe una asociación positiva entre las categorías sin confianza y abstencionistas, 
disminuyendo conforme se incrementa la confianza en las instituciones en las dos categorías siguientes (confianza baja y media). Caso interesante de analizar es la categoría de confianza alta y abstencionista itinerante que, si bien su participación electoral es itinerante, probablemente sea de tipo coyuntural (ver Tabla 5).

Tabla 5: Índice de confianza en instituciones e índice de participación electoral de los electores en el municipio de Tijuana, Baja California, 2010. Distribución porcentual

\begin{tabular}{|c|c|c|c|c|}
\hline \multirow{2}{*}{$\begin{array}{l}\text { Confianza en } \\
\text { Instituciones }\end{array}$} & \multicolumn{4}{|c|}{ Índice de participación electoral } \\
\hline & Participa & $\begin{array}{l}\text { Abstencionista } \\
\text { itinerante }\end{array}$ & $\begin{array}{l}\text { Abstencionista } \\
\text { constante }\end{array}$ & Total \\
\hline Sin confianza & 69.8 & 20.6 & 9.5 & 100.0 \\
\hline Confianza baja & 72.8 & 11.2 & 16.1 & 100.0 \\
\hline Confianza media & 79.4 & 13.1 & 7.5 & 100.0 \\
\hline Confianza alta & 74.4 & 19.5 & 6.1 & 100.0 \\
\hline Total & 75.9 & 15.1 & 9.0 & 100.0 \\
\hline
\end{tabular}

Fuente: Elaboración propia con base en los datos de la Encuesta sobre cultura política y conducta electoral de los ciudadanos residentes en el municipio de Tijuana, Baja California, febrero 2010.

El régimen democrático de alguna manera sustenta que los representantes políticos elegidos para los puestos públicos deben tomar en cuenta los intereses de los ciudadanos y que estos se vean reflejados en la toma de decisiones. Los electores entrevistados, independientemente de la categoría en que estén ubicados en el índice de participación electoral, se inclinan de una regular a una mala representación de los intereses ciudadanos. Siendo más críticos los abstencionistas constantes. Esta variable sí tiene un valor significativo para abstenerse o votar en los procesos electorales (ver Tabla 6).

Tabla 6: Grado de representación de los intereses de los ciudadanos por parte de los partidos políticos e índice de participación electoral de los electores en el municipio de Tijuana, Baja California, 2010. Distribución porcentual

\begin{tabular}{|c|c|c|c|c|c|}
\hline \multirow{2}{*}{$\begin{array}{l}\text { Índice de participación } \\
\text { electoral }\end{array}$} & \multicolumn{5}{|c|}{$\begin{array}{l}\text { Representación de los intereses de los ciudadanos } \\
\text { por parte de los partidos políticos }\end{array}$} \\
\hline & Muy bien & Bien & Regular & Mal & Total \\
\hline Participa & 0.3 & 21.5 & 56.5 & 21.7 & 100.0 \\
\hline Abstencionista itinerante & 0.7 & 40.1 & 24.2 & 34.9 & 100.0 \\
\hline Abstencionista constante & & 2.4 & 55.3 & 42.3 & 100.0 \\
\hline Total & 0.3 & 22.6 & 51.5 & 25.6 & 100.0 \\
\hline
\end{tabular}

Fuente: Elaboración propia con base en los datos de la Encuesta sobre cultura política y conducta electoral de los ciudadanos residentes en el municipio de Tijuana, Baja California, febrero 2010. 
En el tema de la democracia, se retoma la experiencia y método utilizado por Lechner (2002) en una encuesta aplicada en Chile para captar cuál es la idea que tienen los ciudadanos de ella. El resultado de la combinación del índice de la participación electoral y la concepción de la democracia, refleja que los electores entrevistados que participan electoralmente tienen una visión de la democracia consensual y procedimental propia de la teoría de la democracia. En contraparte los abstencionistas, ya sea el itinerante o constante, tiene la idea de la democracia como un instrumento utilitarista desvinculado del lazo social (ver Tabla 7).

Tabla 7: Ejemplo para explicar a un niño qué es la democracia e índice de participación electoral de los electores en el municipio de Tijuana, Baja California, 2010. Distribución porcentual

\begin{tabular}{|c|c|c|c|c|}
\hline \multirow{2}{*}{ Idea de la democracia } & \multicolumn{4}{|c|}{ Índice de participación electoral } \\
\hline & Participa & $\begin{array}{l}\text { Abstencionista } \\
\text { itinerante }\end{array}$ & $\begin{array}{l}\text { Abstencionista } \\
\text { constante }\end{array}$ & Total \\
\hline $\begin{array}{l}\text { Juego al azar donde muchos } \\
\text { juegan y pocos ganan. }\end{array}$ & 7.9 & 39.2 & 0.0 & 11.9 \\
\hline $\begin{array}{l}\text { Un supermercado, en donde } \\
\text { cada quien saca lo que necesita. }\end{array}$ & 22.6 & 34.8 & 58.1 & 27.7 \\
\hline $\begin{array}{l}\text { Juego de futbol, en donde hay } \\
\text { reglas iguales para todos. }\end{array}$ & 25.8 & 2.0 & 32.5 & 22.8 \\
\hline $\begin{array}{l}\text { Como un barco, en donde todos } \\
\text { necesitan jalar parejo. }\end{array}$ & 43.7 & 24.0 & 9.4 & 37.6 \\
\hline Total & 100.0 & 100.0 & 100.0 & 100.0 \\
\hline
\end{tabular}

Fuente: Elaboración propia con base en los datos de la Encuesta sobre cultura política y conducta electoral de los ciudadanos residentes en el municipio de Tijuana, Baja California, febrero 2010.

Se suma a la anterior, la idea que el ciudadano pueda tener del voto; aspecto fundamental en cultura política del ciudadano. Para captar esta idea, se formularon cinco oraciones, las tres primeras tiene que ver con los principios teóricos de la democracia y las dos últimas buscan captar a los ciudadanos que utilizan al voto como forma de expresión (evaluación) hacia el funcionamiento del sistema político (ver Tabla 8).

Como resultado de la combinación del índice de participación electoral y la idea del voto, se observa que entre $50 \%$ y $60 \%$ de los electores entrevistados lo identifican como un derecho y un deber ciudadano. En el subgrupo de los abstencionistas itinerantes, existe $26.6 \%$ de ellos que tienen la concepción del voto como forma de expresión hacia el funcionamiento del sistema político. Para los abstencionistas constantes esta misma idea la tienen en promedio $23.6 \%$ de ellos (ver Tabla 8 ). 
Tabla 8: Concepción del voto e índice de participación electoral de los electores en el municipio de Tijuana, Baja California, 2010. Distribución porcentual

\begin{tabular}{|c|c|c|c|c|}
\hline \multirow{2}{*}{ Concepción del voto } & \multicolumn{4}{|c|}{ Tipo de participación electoral } \\
\hline & Participa & $\begin{array}{l}\text { Abstencionista } \\
\text { itinerante }\end{array}$ & $\begin{array}{l}\text { Abstencionista } \\
\text { constante }\end{array}$ & Total \\
\hline $\begin{array}{l}\text { A la vez es un derecho y un } \\
\text { deber. }\end{array}$ & 58.9 & 62.5 & 57.1 & 59.3 \\
\hline $\begin{array}{l}\text { Es para elegir a nuestros } \\
\text { representantes. }\end{array}$ & 23.4 & 11.0 & 14.4 & 20.7 \\
\hline Es para participar en política. & 2.8 & & 4.9 & 2.6 \\
\hline $\begin{array}{l}\text { Es la única manera de } \\
\text { opinar sobre lo que hace el } \\
\text { gobierno. }\end{array}$ & 6.1 & 12.4 & 18.9 & 8.2 \\
\hline $\begin{array}{l}\text { Es la mejor forma de } \\
\text { manifestar inconformidad con } \\
\text { el gobierno. }\end{array}$ & 8.7 & 14.2 & 4.7 & 9.1 \\
\hline No sabe. & 0.1 & & & 0.1 \\
\hline Total & 100.0 & 100.0 & 100.0 & 100.0 \\
\hline
\end{tabular}

Fuente: Elaboración propia con base en los datos de la Encuesta sobre cultura política y conducta electoral de los ciudadanos residentes en el municipio de Tijuana, Baja California, febrero 2010.

Finalmente, la participación electoral de los ciudadanos residentes en el municipio será diferenciada según el tiempo de vivir en la ciudad. Los datos que arrojó la encuesta señalan que los ciudadanos que acaban de llegar al municipio ( 1 a 5 años), es el grupo que menos vota $(70.5 \%)$ en comparación con los otros inmigrantes y los nativos, pero también un buen porcentaje es itinerante (29.5\%). Pudiera interpretarse como un periodo de adaptación al contexto durante el cual no votan en principio, pero sí eventualmente. En cuanto más tiempo de residencia transcurre, los migrantes empiezan a votar un poco más $(80.7 \%$ y $79.2 \%)$ que los nativos $(73.1 \%)$. Sin embargo, los inmigrantes que tienen entre seis y diez años de residir presentan porcentajes mayores de abstencionistas itinerantes que los nativos, es decir un voto más coyuntural. Mientras que los migrantes que tienen más de diez años de residir en Tijuana son más abstencionistas constantes que los nativos, $11.4 \%$ y $9.7 \%$ respectivamente, dato que abona a la teoría del grado de apropiación de la ciudad (ver Figura 4). 
Figura 4: Índice de participación electoral según tiempo de residencia de los electores en el municipio de Tijuana, Baja California, 2010. Distribución porcentual

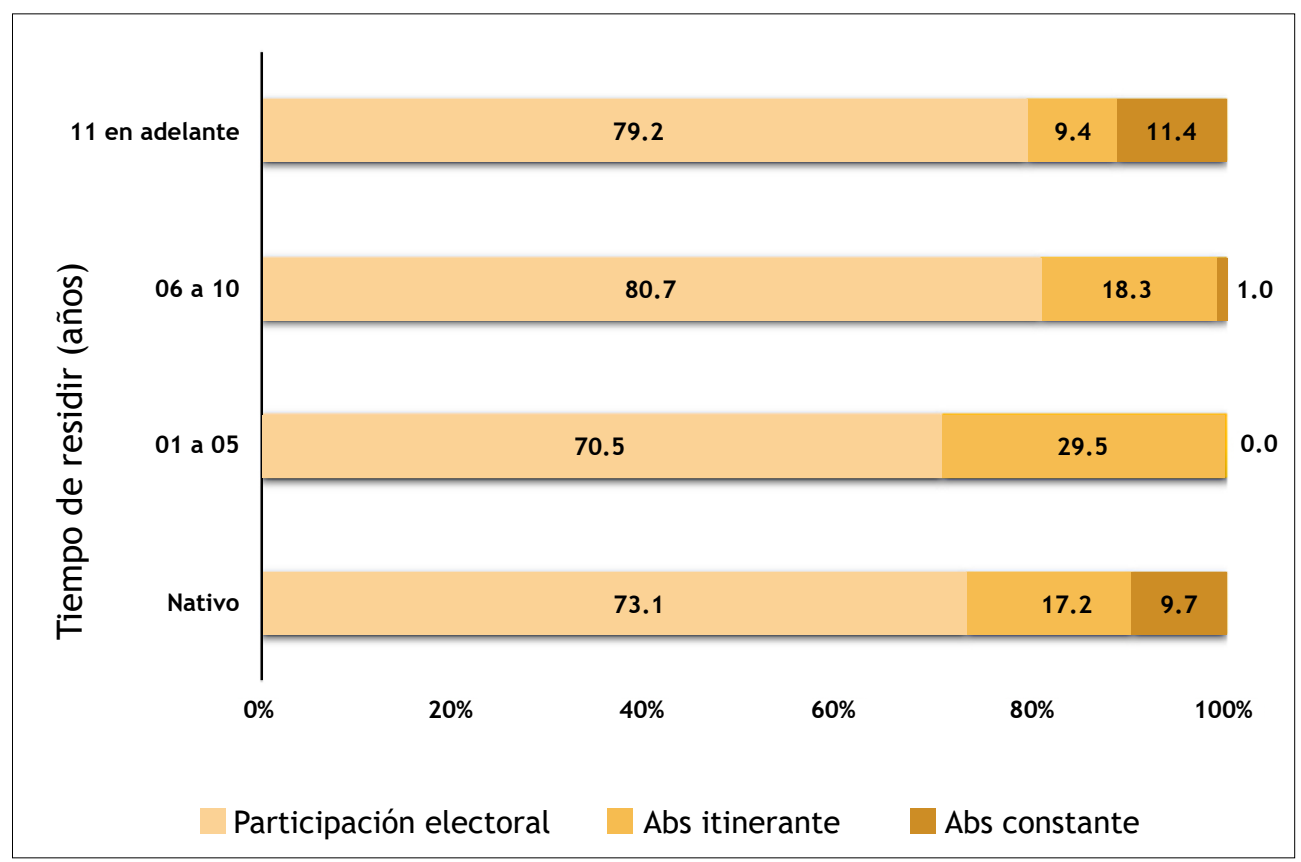

Fuente: Elaboración propia con base en los datos de la Encuesta sobre cultura política y conducta electoral de los ciudadanos residentes en el municipio de Tijuana, Baja California, febrero 2010.

En relación con el último tipo de análisis, se hace uso de un modelo de regresión logística y los datos que arroja la Encuesta para estimar los elementos sociodemográficos asociados a la probabilidad de que una persona sea abstencionista. El modelo analiza la relación que existe entre un conjunto de variables sociodemográficas, de cultura política y la probabilidad de que un ciudadano sea abstencionista (ver Tabla 9).

Tabla 9: Modelo de regresión logística para la probabilidad de ser abstencionista. Electores en el municipio de Tijuana, Baja California, 2010

\begin{tabular}{|c|c|c|c|c|}
\hline & B & S.E. & Sig. & $\operatorname{Exp}(B)$ \\
\hline Edad & -0.058 & 0.017 & 0.001 & 0.943 \\
\hline Escolaridad & -1.893 & 0.425 & 0.000 & 0.151 \\
\hline Simpatía por un partido político & 0.762 & 0.352 & 0.030 & 2.143 \\
\hline $\begin{array}{l}\text { Evaluación del desempeño del } \\
\text { partido político }\end{array}$ & 0.838 & 0.361 & 0.020 & 2.311 \\
\hline Constant & 1.741 & 0.850 & 0.041 & 5.705 \\
\hline
\end{tabular}

Fuente: Elaboración propia con base en los datos de la Encuesta sobre cultura política y conducta electoral de los ciudadanos residentes en el municipio de Tijuana, Baja California, febrero 2010. 
Entre los principales resultados que se obtuvieron a partir del modelo antes referido se encuentran los siguientes:

a) Edad. A mayor edad se observa una menor probabilidad de ser abstencionista. Por cada año de edad, se reduce en aproximadamente $5 \%$ la probabilidad de ser abstencionista.

b) Nivel educativo. Las personas con nivel de estudios de secundaria, preparatoria y profesional tienen menor probabilidad de ser abstencionistas que quienes tienen solo primaria (alrededor de $85 \%$ menos probable).

c) Simpatía por un partido político. Los que simpatizan con el PAN tienen poco más del doble de probabilidad de ser abstencionistas que los que simpatizan con cualquier otro partido político. Pero también los que no simpatizan con ningún partido tienen más posibilidad de ser abstencionistas.

d) Evaluación del gobierno local. Entre menos favorable sea la evaluación de los ciudadanos sobre el desempeño del gobierno local tienen mayores posibilidades de ser abstencionistas.

e) Evaluación de partidos políticos. Si la evaluación que los ciudadanos realizan al desempeño de los partidos políticos es menos favorable existen mayores posibilidades de ser abstencionistas.

f) Los jóvenes con alta escolaridad, que no simpatizan con un partido político, que hacen una evaluación mala del gobierno local, y también del desempeño de los partidos políticos, son quienes tienen más propensión a ser abstencionistas.

\section{Conclusiones}

Entre los principales resultados de este estudio sobresale, desde el ámbito de la estadística electoral, en primer lugar, la relativamente alta participación electoral a fines de los años ochenta en el municipio y su declive considerable en posteriores elecciones, hasta casi $70 \%$ en las elecciones locales de 2016.

Respecto al lugar de residencia de los abstencionistas y la frecuencia con que realizan esta actitud política, los resultados indican que estamos frente a un problema real y sustantivo. Pues, si bien la abstención se presenta en todo el municipio, su concentración y su incremento en el periodo que va de 2004 a 2016 ha sido diferente para cada distrito electoral, en donde sobresale el distrito xII como un área políticaelectoral con presencia histórica de la abstención y con marcada tendencia creciente de este fenómeno.

En relación con los principales resultados del perfil sociopolítico del elector fronterizo, se destaca la presencia de tres grupos: a) los que participan, $b$ ) los abstencionistas itinerantes, y c) los abstencionistas constantes. Ello nos permitió identificar diferencias sustantivas, a la luz de la elaboración de índices y de un modelo de regresión logística. Es decir, la cultura política y la actitud electoral tiene matices claramente identificados entre los electores nativos y los inmigrantes según su tiempo de residencia en el municipio.

Los principales rasgos de la cultura política de los abstencionistas son los siguientes: 
a) Existen diferencias importantes entre los resultados electorales en los comicios federales y locales. Desde la asistencia a las urnas hasta la perspectiva que se tiene del voto.

b) Los abstencionistas tienen una idea del voto como forma de expresión y de evaluación respecto del funcionamiento del sistema político.

c) Los abstencionistas nativos tiene una idea de la democracia en un término más instrumental y utilitarista.

d) Los electores inmigrantes que tienen entre uno y cinco años de residencia en Tijuana se autoubicaron en la derecha ideológica y participan electoralmente o son abstencionistas itinerantes.

e) Existe una asociación significativa positiva entre la variable "sin confianza en las instituciones" y la abstención.

f) Existe una asociación positiva entre la evaluación del desempeño gubernamental, la representatividad de los partidos políticos y la abstención.

Finalmente, es importante destacar que en este estudio se consideraron dos grupos de control, el de los nativos y de los ciudadanos inmigrantes dada la perspectiva histórica del fenómeno migratorio en el municipio y el complejo contexto de una sociedad fronteriza. Que en palabras de Espinoza y Ham (2011) "las oscilaciones en su incremento demográfico (de Tijuana) están en función de los factores que atraen, expulsan y condicionan la composición y las características tanto de la población migrante como de la ya asentada" (Espinoza y Ham, 2011, p. 177). Factores identificados en algunos puntos, como la diferencia entre la actitud política de los nativos y no nativos en su intervención en la esfera pública; por ejemplo, la participación ciudadana más intensa entre los inmigrantes que los nativos y, la abstención constante entre los inmigrantes con más de 10 años de residencia en el municipio.

En este sentido, es necesario continuar estudiando la participación electoral de los inmigrantes residentes en el municipio por tiempo de residencia y los factores fronterizos que lo impactan, como profundizar más en al grado de apropiación del territorio o de la ciudad, con el objetivo de identificar las variables que intervienen en la construcción de la ciudadanía o los factores que están inhibiéndola.

\section{Referencias}

Acosta, F., Solís, M. y Alonso, G. (2012). Grado de apropiación de la ciudad y percepciones sobre la calidad de vida en ciudades de la frontera norte de México. Cofactor, 3(6), 11-42.

Alcántara, M. (2008). La escala de la izquierda. La ubicación ideológica de presidentes y partidos de izquierda en América Latina. Nueva Sociedad, (217), 72-85.

Alcubilla, E. (s.f.). Abstencionismo electoral. Diccionario Centro de Asesoría y Promoción Electoral. Recuperado de https://www.iidh.ed.cr/multic/WebServices/Files.ashx?fileID $=2545$ 
Barquín, M. (2003). Elector. En Instituto Interamericano de los Derechos Humanos (edit.), Diccionario Electoral (pp. 163-165). San José, Costa Rica: Centro de Asesoría y Promoción Electoral; Instituto Interamericano de los Derechos Humanos.

Consejo Nacional de Población (Conapo). (2012). Cuadro 5.2. Zona metropolitana de Tijuana: Población, tasa de crecimiento y densidad media urbana, 1990-2010. Recuperado de http://www.conapo.gob.mx/work/models/CONAPO/zonas_metropolitanas_2010/cuadros/ZM_02.xlsx

Coutigno, A. C. (2012) . ... Los sueños democráticos no entran en las urnas. La abstención electoral en el municipio de Tijuana, Baja California 1989-2010 (Tesis doctoral). Centro de Investigaciones y Estudios Superiores en Antropología Social-Occidente, Guadalajara, Jalisco.

Encuesta sobre cultura politica y conducta electoral de los ciudadanos residentes en el municipio de Tijuana, Baja California, febrero 2010. Baja California, México.

Espinoza, V. A. (Coord.). (2007). Baja California, 2004: Abstencionismo y alternancia política. En V. Espinoza (coord.), Las rutas de la democracia. Elecciones locales en México (pp. 123-140). México: Centro de Estudios de Política Comparada.

Espinoza, P. y Ham, R. (2011). Un siglo de crecimiento demográfico en Baja California. En D. Piñera y J. Carrillo (Coords.). Baja California a 100 años de la Revolución Mexicana: 1910-2010 (pp. 177-194). Baja California, México: El Colegio de la Frontera Norte; Universidad Autónoma de Baja California.

Gómez, S. (2009). ¿Cuántos votos necesita la democracia? La participación electoral en México 1961-2006. México: Instituto Federal Electoral.

Instituto Estatal Electoral de Baja California (ІеЕвC). (2016). Resultados electorales. Recuperado de http://www.ieebc.mx/resultados.html

Instituto Nacional Electoral (INE). (2016). Estadísticas y resultados electorales. Recuperado de http://www.ine.mx/archivos3/portal/historico/contenido/Historico_de_Resultados_Electorales/

Merino, M. (1995). La participación ciudadana en la democracia. Cuadernos de divulgación de la cultura democrática, 4.

Nohlen, D. (2003). Ampliación de la participación política y reducción del abstencionismo: Ejes de una cultura democrática y una nueva ciudadanía para el siglo XXI. Ponencia presentada en la Xvir Conferencia Protocolo de Tikal, San José, Costa Rica.

Nohlen, D. (2004). La participación electoral como objeto de estudio. Revista Elecciones, (3), 137-157.

Lechner, N. (2002). Análisis de encuestas de cultura política. Ponencia presentada en el Coloquio para el Análisis de Encuestas sobre Cultura Política y Prácticas Ciudadanas, México, Distrito Federal.

Peschard, J. (2001). La participación ciudadana en la democracia. Cuadernos de divulgación de la cultura democrática, 2.

Raventós, C. (2003). Abstención: conceptos y alcances, problemas asociados y posibilidades de superación. Ponencia presentada en la XviI Conferencia del Protocolo de Tikal, San José, Costa Rica.

Salazar, R. y Temkin, B. (2007). Abstencionismo, escolaridad y confianza en las instituciones. Las elecciones federales de 2003 en México. Política y Gobierno, xVI(1), $5-42$. 
Sartori, G. (2007). ¿Qué es la Democracia? México: Taurus.

Somuano, Ma. F., Nieto, F. y Zaremberg, G. (2014). Ciudadanía en México. La importancia del contexto. Recuperado de http://portalanterior.ine.mx/archivos2/s/ DECEYEC/EducacionCivica/La-Importancia-del-contexto.pdf

Zenteno, R. M. (1995). Del rancho de la Tía Juana a Tijuana: Una breve historia de desarrollo y población en la frontera norte de México. Estudios Demográficos y Urbanos, 10(1), 105-132.

Ana Claudia Coutigno Ramírez

Mexicana. Doctora en Ciencias Sociales con especialidad en Estudios de Políticas Sociales y Ciudadanía por el Centro de Investigaciones y Estudios Superiores en Antropología Social. Forma parte del personal académico de El Colegio de la Frontera Norte, pertenece al Sistema Nacional de Investigadores Nivel I y es miembro de la Sociedad Mexicana de Estudios Electorales. Líneas de investigación: ciudadanía, cultura política y procesos electorales locales y regionales. Publicación reciente: (2016). Baja California elecciones 2016. La tendencia electoral desde los municipios y las secciones electorales. En Los estados 2016. Nuevos equilibrios regionales. UNAM. 